\title{
Three-Dimensional Echocardiography in Evaluating LA Volumes and Functions in Diabetic Normotensive Patients without Symptomatic Cardiovascular Disease
}

\author{
Mohamed Hamza $\left(\mathbb{D},{ }^{1}\right.$ Ahmed Mamdouh, ${ }^{1}$ Dina Ezzeldin, ${ }^{1}$ Adnan Tawfik, \\ and Ahmed Nayel ${ }^{1}$ \\ ${ }^{1}$ Department of Cardiology, Ain Shams University, Cairo, Egypt \\ ${ }^{2}$ Kalar General Hospital, Sulaimania, Iraq \\ Correspondence should be addressed to Mohamed Hamza; matef77@hotmail.com
}

Received 20 January 2020; Revised 18 June 2020; Accepted 8 July 2020; Published 19 August 2020

Academic Editor: Alberto Caggiati

Copyright (c) 2020 Mohamed Hamza et al. This is an open access article distributed under the Creative Commons Attribution License, which permits unrestricted use, distribution, and reproduction in any medium, provided the original work is properly cited.

\begin{abstract}
Background. Cardiovascular complications are the most serious threat to diabetic patients. Associated metabolic and microvascular changes are the main cause of cardiac function affection, and the earliest cardiac change is diastolic dysfunction. Assessment of LA function changes is a key to determine early heart damage of diabetic patients. Objectives. To evaluate the effect of diabetes mellitus on left atrial volumes and functions by using real-time 3-dimensional echocardiography in normotensive patients free from cardiovascular disease. Methods. The study included 110 individuals, 50 controls and 60 patients with diabetes mellitus, 30 patients with type 1 diabetes mellitus and 30 patients with type 2 diabetes mellitus. 2-dimensional echocardiography was used to assess the LA maximum volume and LA phasic volumes, and LA maximum volume indexed to body surface area were measured by 3D echocardiography. LA functions (LA total stroke volume, LA active stroke volume, and LA active emptying fraction) were obtained from RT3D volumetric analysis. Results. The results of the analysis revealed that type 2 diabetes mellitus showed enlarged $V_{\max }, V_{\min }$, and LAVi with an increased LA total stroke volume and decreased active emptying fraction, while type 1 diabetics showed only decreased in active emptying fraction. The LA maximum volume indexed to body surface area (LAVi) was significantly higher in type 2 diabetic patients as compared to normal controls which was $23.55 \pm 3.37 \mathrm{ml} / \mathrm{m}^{2}$ versus 20.30. Conclusion. Patients with type 2 diabetes mellitus have an increased LA volume with impaired compliance and contractility, while patients with type 1 diabetes mellitus have only impaired contractility compared to nondiabetic subjects.
\end{abstract}

\section{Background}

The quantification of the cardiac chamber size and function is the cornerstone of cardiac imaging, with echocardiography being the most commonly used noninvasive modality because of its unique ability to provide real-time images of the beating heart with high temporal and spatial resolution, combined with its availability and portability [1]. [2]

Using the advanced echocardiographic techniques, such as strain (S), Doppler, speckle tracking, and 3D echocardiography, we are able to recognize early atrial dysfunction, before clinical manifestations and earlier than standard echocardiographic parameters. [3]

Cardiovascular complications are the most serious threat of diabetes to diabetic patients. The associated metabolic and microvascular changes are the main cause of cardiac function affection, and the earliest cardiac change of diabetes is diastolic dysfunction. [4] Assessment of LA function changes is a key to determine early heart damage of diabetic patients [5].

Two-dimensional echocardiography has been the most commonly used diagnostic modality for assessing the LA size and function in daily clinical situations. However, the 
measurement of the LA volume is difficult due to its complex shape.

Several methods which use various geometric assumptions about the atrial shape have been developed for assessing the LA volume, such as the biplane area length (AL), the biplane modified Simpson, and the prolate ellipse methods [6].

A volumetric system developed at Duke University enabled the first real-time acquisitions. Consequently, RT3DE was used in recent studies to evaluate the LA size and/or function in various diseases [7].

\section{Aim}

The study is aimed at evaluating the effect of diabetes mellitus on left atrial volumes and functions by using real-time threedimensional echocardiography in normotensive patients free of symptomatic cardiovascular disease.

\section{Methods}

The study was a case control study and included 110 individuals, 50 normal healthy subjects regarded as control and 60 patients with diabetes mellitus, 30 patients with type 1 diabetes mellitus and 30 patients with type 2 diabetes mellitus.

The inclusion criteria were age above 18 years, normal blood pressure, left ventricular ejection fraction $\geq 50 \%$ (modified Simpson method), sinus rhythm, and with no history of previous cardiac symptoms.

The diabetic patients included were already diagnosed as either type 1 or type $2 \mathrm{DM}$, were taking their antidiabetic medication, and were regularly visiting the diabetes clinic for follow-up.

Patients with hypertension (previously diagnosed, on antihypertensive medications, or measured BP >140/90), structural heart disease (valvular and congenital heart disease, LVH, and impaired LV systolic functions), ischemic heart disease (by history of revascularization or documented evidence of ischemia), and arrhythmias and patients with chronic kidney disease and chronic obstructive lung diseases were all excluded from the study.

The control group consisted of 50 healthy individuals matched for age and sex with the diabetic patients.

After a written informed consent, all study participants had meticulous clinical assessment (history and physical examination) with calculation of the estimated BSA $\left(\mathrm{cm}^{2}\right)$, ECG, and echo study.

All echocardiographic examinations were performed by the same echocardiographer, by using IE33, Philips machine, with digital storage software for offline analysis [8].

LV internal dimensions, wall thickness, and LA diameter were obtained from the parasternal long-axis view [9], and LV ejection fraction was calculated from apical four-chamber and two-chamber views using Simpson's biplane method [10] Conventional pulsed Doppler imaging of mitral inflow was recorded from apical 4-chamber view to measure the $E$ and $A$ waves and calculate the $E$ / $A$ ratio [11]. LA volumes were measured using Simpson's biplane method of discs with the LA appendage and pul- monary veins excluded from the tracing using fourchamber and two-chamber views [12].

Subsequently, all subjects performed three-dimensional echocardiographic evaluations.

Individuals were instructed to hold their breath at the time of image acquisition, and images were coupled with ECG recording. ${ }^{(197)}$

The real-time three-dimensional echocardiography (RT3DE) was used to obtain the left atrial volume.

An IE33 echocardiography device, with an X3 (1-3 MHz) matrix transducer by the same echocardiographer was used to acquire the full volume, real-time pyramidal volumetric data for four consecutive cardiac cycles. To ensure the inclusion of the entire volume of the LA and LV inside the pyramidal volume, data were acquired using the wide-angle mode, thus acquiring four wedge-shaped subvolumes for a period of 5 seconds of apnea [13].

The echocardiographic 3D data were digitally stored and analyzed using the QLAB-Philips software (version 6.0; Philips Medical Systems). The three-dimensional echocardiographic image analysis was based on the apical window and semiautomatic tracing of the endocardial borders (Figure 1). [14]

The analysis of the left atrium was performed using MPR mode by marking four points on the atrial surface of the mitral annulus, septal, lateral, anterior, and inferior, and a fifth point in the left atrial roof. Subsequently, the endocardial surface was automatically outlined and could be visualized from different views [15].

Manual modifications were made to correct the automatic tracing when necessary, and then, the software generates a variation curve of the LAV throughout the cardiac cycle [16]. The LA appendage and the pulmonary vein confluence were excluded from the LA tracings, and finally, the dynamic LA polyhedron model was obtained [17].

The $\mathrm{LAV}_{\max }$ was considered the peak of the curve, and the value was indexed by the body surface area (LAVi) [18]..

The following left atrial volumes were measured: LA minimum volume $\left(\mathrm{LAV}_{\min }\right)$ and $\mathrm{LA}$ end-diastolic volume measured at the first frame after mitral valve closure (Figure 2) [19], LA maximum volume $\left(\mathrm{LAV}_{\max }\right)$ and LA end-systolic volume measured one frame before mitral valve opening (Figure 3) [19], and LA volume before contraction $\left(V_{\text {preA }}\right)$ : the last frame before mitral valve reopening or at the time of P wave on ECG (Figure 4) [19].

From these volumes, the following indices of LA function were calculated: LA total stroke volume (LASV): $\mathrm{LAV}_{\max }$ $\mathrm{LAV}_{\text {min }}$, LA active stroke volume (ASV): $V_{\text {preA }}-V_{\min }$, and LA active emptying fraction (AEF): ASV $/ V_{\text {preA }} \times 100$ [20].

\section{Statistical Analysis}

Data were collected, revised, coded, and entered to the Statistical Package for Social Science (IBM SPSS) version 20. Qualitative data were presented as number and percentages while quantitative data were presented as mean, standard deviations, and ranges. 


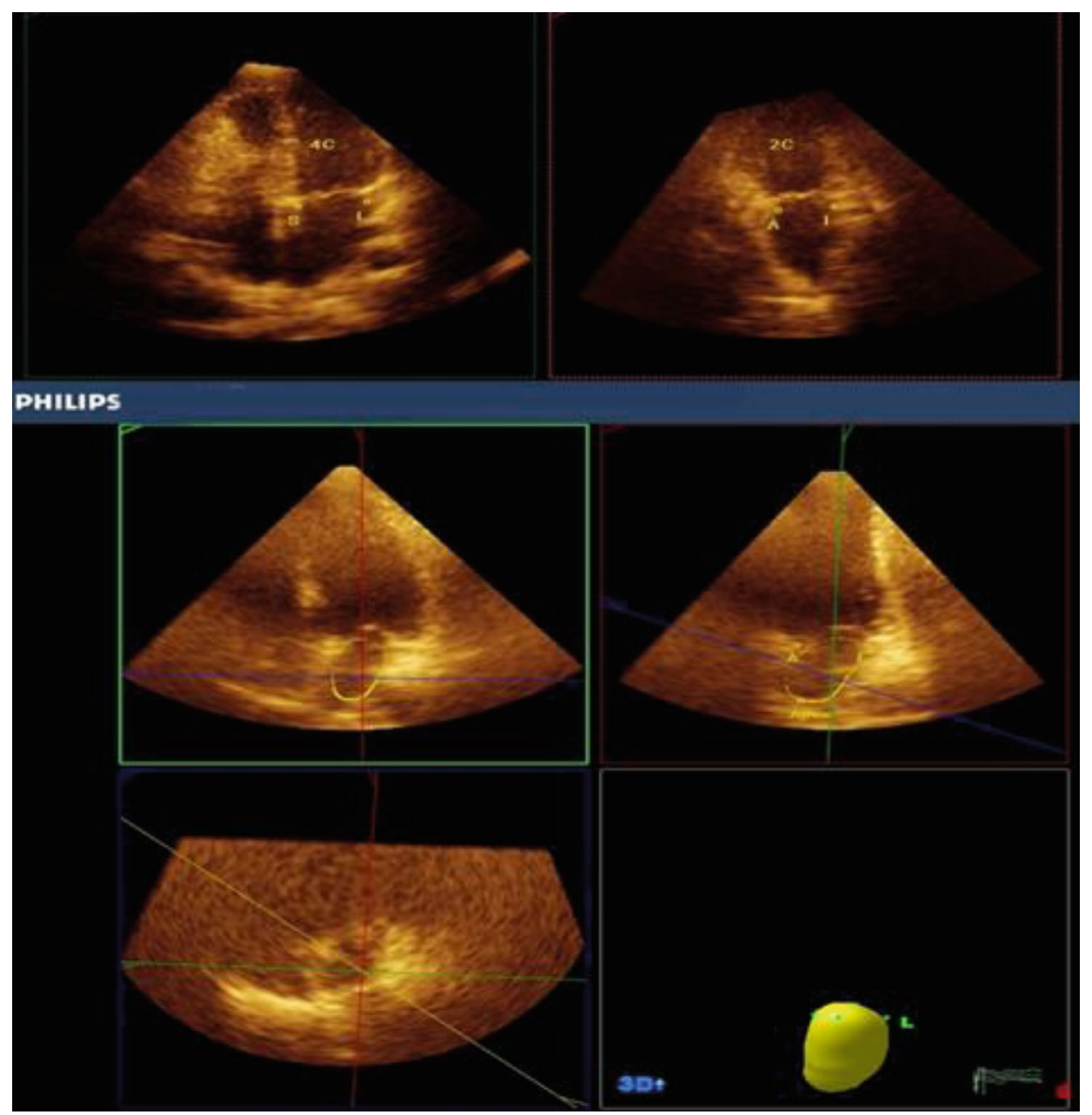

Figure 1: Semiautomatic LA border tracing by marking $(\bullet)$ at 4 mitral annular points (lateral, septal, inferior, and anterior) and an atrial superior dome point opposite the annulus A. The automatic border tracing is then shown by the software.

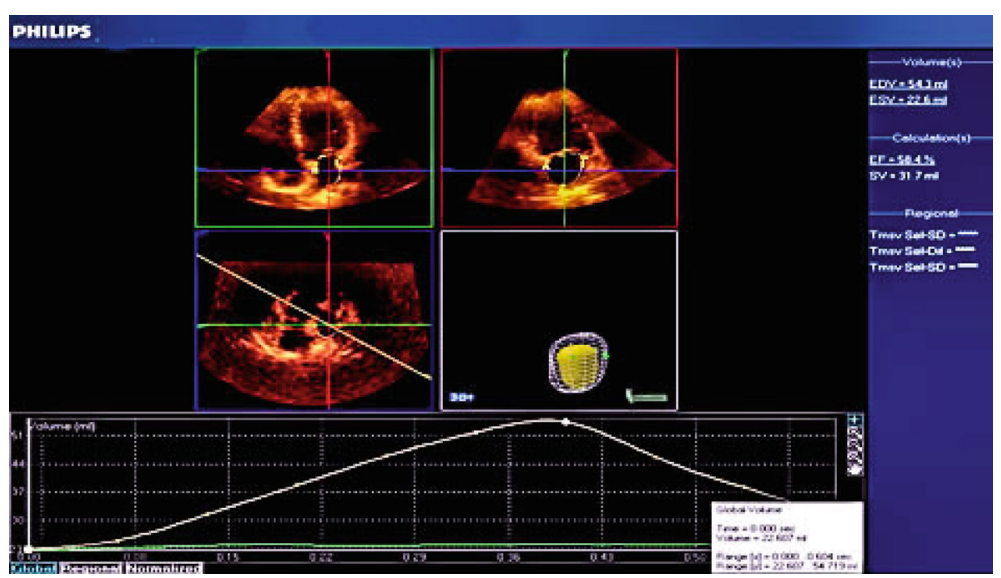

FIGURE 2: Left atrial volume by real-time three-dimensional echocardiography at the end diastole $\left(\mathrm{LAV}_{\min }\right)$.

The comparison between two groups with qualitative data were done by using the chi-squared test, and/or the Fisher exact test was used instead of the chi-squared test when the expected count in any cell was found less than 5 .

The comparison between two independent groups regarding quantitative data with parametric distribution was done by using the independent $t$-test, while comparison between more than two groups with quantitative data was done by using one-way analysis of variance (ANOVA).

Spearman correlation coefficients were used to assess the relation between two quantitative parameters in the same group. 


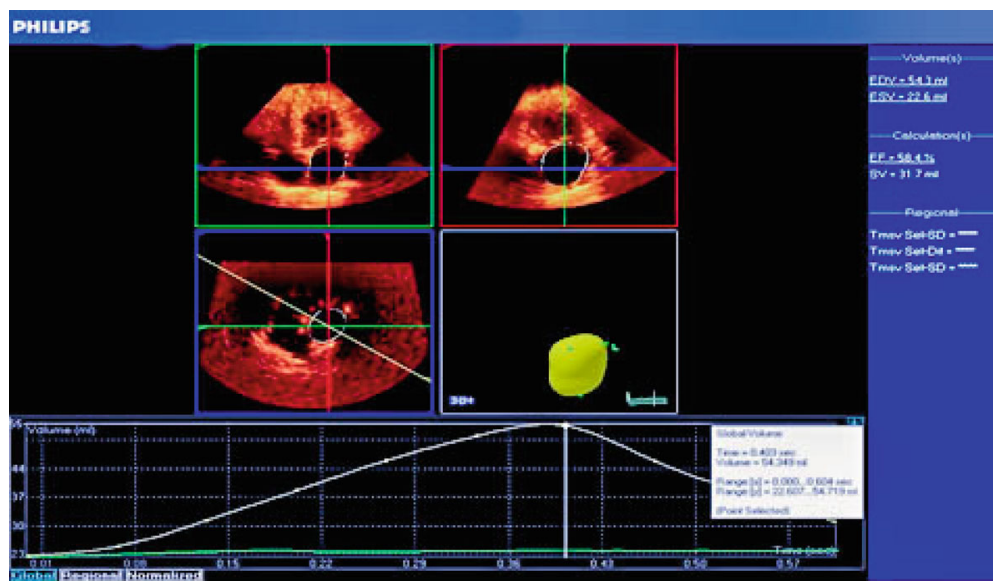

FIgURE 3: Left atrial volume by real-time three-dimensional echocardiography at the end systole ( $\left.\mathrm{LAV}_{\max }\right)$.

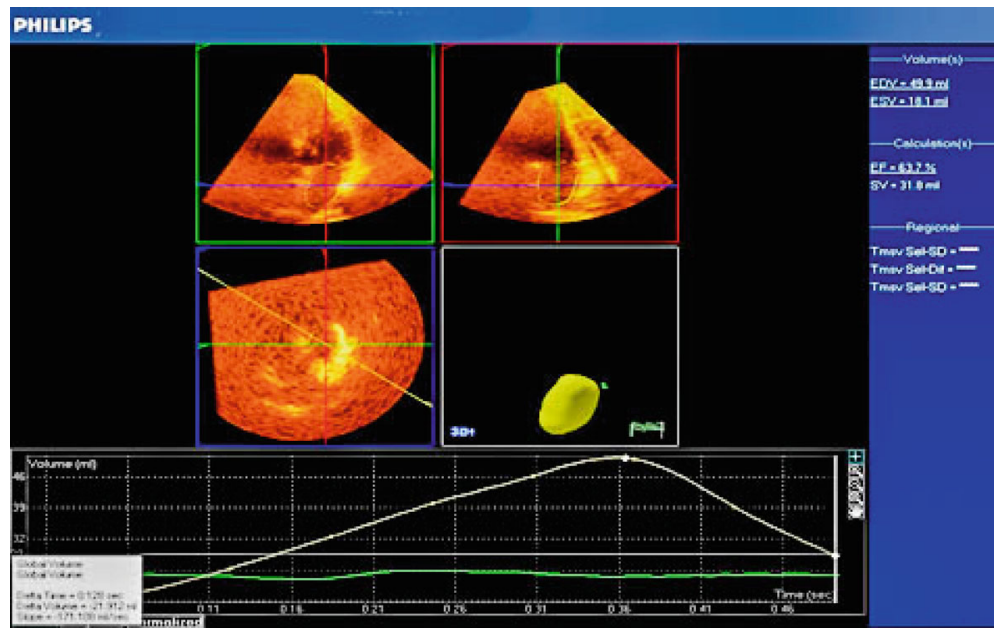

FIgURE 4: Left atrial volume by real-time three-dimensional echocardiography at last frame before mitral reopening $\left(V_{\text {preA }}\right)$.

The confidence interval was set to $95 \%$, and the margin of error accepted was set to $5 \%$. So, a $P$ value less than 0.05 was considered significant.

\section{Results}

This study included 110 individuals, 50 normal healthy subjects and 60 patients with diabetes mellitus, 30 patients with type 1 diabetes mellitus and 30 patients with type 2 diabetes mellitus. Patients were consecutively recruited from the diabetic clinic of Ain Shams University hospital. Baseline demographic data including age and sex are listed in Table 1.

The duration of diabetes in type 1 diabetic patient group ranged from 4 to 26 years with a mean of $12.60 \pm 6.32$, and the duration of diabetes in type 2 diabetic patients ranged from 1 to 20 years with a mean of $10.20 \pm 6.19$. There was no significant difference in the duration of diabetes in both subgroups $(P>0.05)$.

Regarding LV dimensions, wall thickness, and LV systolic functions, there was no statistically significant difference between the 3 groups included in the study (control subjects and type 1 and type 2 diabetic patients) (Table 2 ).
5.1. Left Atrial Dimensions and Area by 2D Echocardiography. The LA area was larger in the diabetic group $(P<0.05)$ while the LA length and diameter showed no statically significant difference between control subjects and diabetic patients (Table 3).

5.2. Left Atrial Volume by Two-Dimensional Echocardiography. The patient group shows a significantly larger left atrial volume in comparison to control group $(P<0.005)$ (Table 4$)$.

After dividing the patient group into diabetic subgroups (type 1 and 2), there was no statistically significant difference between controls and diabetic type 1 regarding LA volumes but type 2 diabetic patients showed a statistically significant higher LA volumes when compared to controls.

There was a statistically significant difference between both study groups regarding mitral $E / A$ ratio and $A$ velocity $(P<0.05)$.

Regarding the mitral inflow velocity $(E / A)$ ratio, there was no significant difference between control subjects and type 1 diabetics, but on the other hand, there was a statistically significant difference between controls and type 2 diabetes patients. 
TABle 1: Baseline demographic data.

\begin{tabular}{|c|c|c|c|c|}
\hline & & Control group & D1 group & D2 group \\
\hline \multirow{2}{*}{ Age (year) } & Mean \pm SD & $33.63 \pm 8.57$ & $25.53 \pm 5.88$ & $51.97 \pm 9.33$ \\
\hline & Range & $18-58$ & $18-38$ & $37-70$ \\
\hline \multirow{2}{*}{ Sex } & Female & $17(34 \%)$ & $24(80 \%)$ & $22(73.3 \%)$ \\
\hline & Male & $33(66 \%)$ & $6(20 \%)$ & $8(26.7 \%)$ \\
\hline \multirow{2}{*}{ Weight $(\mathrm{kg})$} & Mean \pm SD & $74.65 \pm 13.58$ & $61.87 \pm 8.80$ & $72.40 \pm 8.02$ \\
\hline & Range & 50-95 & $45-80$ & 55-90 \\
\hline \multirow{2}{*}{ BMI $\left(\mathrm{kg} / \mathrm{m}^{2}\right)$} & Mean \pm SD & $25.45 \pm 3.26$ & $23.32 \pm 2.90$ & $26.26 \pm 2.79$ \\
\hline & Range & $18.4-29.7$ & $16.5-28.1$ & $20.2-29.4$ \\
\hline \multirow{2}{*}{ Weight (kg) } & Mean \pm SD & $74.65 \pm 13.58$ & $61.87 \pm 8.80$ & $72.40 \pm 8.02$ \\
\hline & Range & 50-95 & $45-80$ & 55-90 \\
\hline \multirow{2}{*}{ Duration of diabetes (year) } & Mean \pm SD & & $12.60 \pm 6.32$ & $10.20 \pm 6.19$ \\
\hline & Range & & $4-26$ & $1-20$ \\
\hline
\end{tabular}

TABLE 2: Comparison between patient and control groups regarding LV dimensions and function.

\begin{tabular}{lcccc}
\hline & & Control group & Patients group & \multicolumn{2}{c}{ Independent $t$-test } \\
& & No. $=50$ & No. $=60$ & $P$ value \\
\hline \multirow{2}{*}{ LVEDd $(\mathrm{mm})$} & Mean \pm SD & $41.68 \pm 4.41$ & $42.29 \pm 4.44$ & 0.755 \\
& Range & $35-51$ & $33-63$ & 0.451 \\
\hline \multirow{2}{*}{ LVESd (mm) } & Mean \pm SD & $29.06 \pm 2.05$ & $28.98 \pm 3.41$ & 0.165 \\
\hline \multirow{2}{*}{ IVS thickness $(\mathrm{mm})$} & Range & $25-34$ & $22-38$ & 3.948 \\
\hline \multirow{2}{*}{ LVEF\% } & Mean \pm SD & $9.09 \pm 0.89$ & $9.68 \pm 0.74$ & 0.001 \\
& Range & $5-10.3$ & $8-10.3$ & 1.378 \\
\hline
\end{tabular}

TABLE 3: Comparison between the control group and the patient group regarding LA area and dimensions by $2 \mathrm{D}$.

\begin{tabular}{|c|c|c|c|c|c|}
\hline & & \multirow{2}{*}{ Control group } & \multirow{2}{*}{ Patient group } & \multicolumn{2}{|c|}{ Independent $t$-test } \\
\hline & & & & $t$ & $P$ value \\
\hline \multirow{2}{*}{ LA area $\left(\mathrm{cm}^{2}\right)$} & Mean \pm SD & $13.34 \pm 2.29$ & $14.05 \pm 2.94$ & \multirow{2}{*}{3.455} & \multirow{2}{*}{0.001} \\
\hline & Range & $10-19.1$ & $11.43-21.2$ & & \\
\hline \multirow{2}{*}{ LA length $(\mathrm{cm})$} & Mean \pm SD & $4.79 \pm 0.63$ & $5.84 \pm 5.11$ & \multirow{2}{*}{0.695} & \multirow{2}{*}{0.489} \\
\hline & Range & $3.7-6.2$ & $3-6.1$ & & \\
\hline \multirow{2}{*}{ LA diameter $(\mathrm{mm})$} & Mean \pm SD & $32.71 \pm 2.68$ & $32.68 \pm 2.85$ & \multirow{2}{*}{1.281} & \multirow{2}{*}{0.203} \\
\hline & Range & $27-39$ & $28-38$ & & \\
\hline
\end{tabular}

We studied 30 cases with diabetes mellitus type 1 in which $16.7 \%$ had left ventricular diastolic dysfunction grade $1(E / A<1)$ and 30 cases with diabetes mellitus type 2 in which 18 cases $(60 \%)$ had left ventricular diastolic dysfunction grade $1(E / A<1)$.

5.3. Left Atrial Volume by 3D Echocardiography. The LA minimum volume, maximum volume, volume before atrial contraction, and LA volume indexed to the BSA were significantly higher in patients with type 2 diabetes $P$ value $<0.05$ while there was no significant difference between control subjects and patients with type 1 diabetes regarding $V_{\min }$, $V_{\text {max }}, V_{\text {preA }}$, and LAVi $(P>0.05)$.(Table 5).

5.4. Left Atrial Function by 3D Echocardiography. Patients with type 1 diabetes showed a significantly lower active 
TABLE 4: Comparison between patient and control groups regarding 2D LA volumes.

\begin{tabular}{|c|c|c|c|c|c|}
\hline & & \multirow{2}{*}{$\begin{array}{c}\text { Control group } \\
\text { No. }=50\end{array}$} & \multirow{2}{*}{$\begin{array}{c}\text { Patients group } \\
\text { No. }=60\end{array}$} & \multicolumn{2}{|c|}{ Independent $t$-test } \\
\hline & & & & $t$ & $P$ value \\
\hline \multirow{2}{*}{ 4C LA volume (ml) } & Mean \pm SD & $29.20 \pm 6.51$ & $33.74 \pm 6.75$ & \multirow{2}{*}{3.57} & \multirow{2}{*}{0.001} \\
\hline & Range & $20-45$ & $12-61$ & & \\
\hline \multirow{2}{*}{ 2C LAV (ml) } & Mean \pm SD & $31.45 \pm 8.15$ & $35.79 \pm 6.98$ & \multirow{2}{*}{3.009} & \multirow{2}{*}{0.003} \\
\hline & Range & $20-50$ & $12-61$ & & \\
\hline \multirow{2}{*}{ 2C LAV (bi plane) (ml) } & Mean \pm SD & $31.85 \pm 7.15$ & $36.17 \pm 6.89$ & \multirow{2}{*}{3.219} & \multirow{2}{*}{0.002} \\
\hline & Range & $24-50$ & $14-61$ & & \\
\hline
\end{tabular}

TABLE 5: Comparison between control group and patient group regarding left atrial volumes by 3D. D1: type 1 diabetes; D2: type 2 diabetes.

\begin{tabular}{lccccrrr}
\hline & & Control group & D1 group & D2 group & P1 & P2 & P3 \\
\hline \multirow{2}{*}{$V_{\text {min }}$} & Mean \pm SD & $12.41 \pm 2.59$ & $12.78 \pm 2.12$ & $17.29 \pm 4.11$ & 0.511 & 0.001 \\
\hline \multirow{2}{*}{$V_{\text {max }}$} & Range & $9-17$ & $9-20$ & $12-30$ & 0.009 \\
\hline \multirow{2}{*}{$V_{\text {preA }}$} & Mean \pm SD & $32.44 \pm 6.81$ & $35.29 \pm 7.64$ & $44.09 \pm 9.44$ & 0.076 & 0.001 \\
\hline \multirow{2}{*}{ LAVi } & Range & $24-50$ & $15-55$ & $34-83$ & 0.001 \\
& Mean \pm SD & $23.68 \pm 6.16$ & $23.02 \pm 4.34$ & $24.53 \pm 2.62$ & 0.600 & 0.514 \\
\hline
\end{tabular}

TABLE 6: Comparison between the control group and the patient subgroup regarding left atrial function by 3D.

\begin{tabular}{|c|c|c|c|c|c|c|c|}
\hline & & Control group & D1 group & D2 group & $\mathrm{P} 1$ & $\mathrm{P} 2$ & P3 \\
\hline \multirow{2}{*}{ LA total stroke volume } & Mean \pm SD & $21.84 \pm 4.04$ & $22.91 \pm 4.88$ & $25.26 \pm 4.14$ & \multirow{2}{*}{0.275} & \multirow{2}{*}{0.001} & \multirow{2}{*}{0.049} \\
\hline & Range & $14-29$ & $17.1-44.2$ & $18-33.5$ & & & \\
\hline \multirow{2}{*}{ LA active stroke volume } & Mean \pm SD & $10.02 \pm 3.0$ & $9.73 \pm 2.15$ & $9.71 \pm 3.71$ & \multirow{2}{*}{0.635} & \multirow{2}{*}{0.665} & \multirow{2}{*}{0.976} \\
\hline & Range & $3.6-16$ & $6.2-17$ & 4-19 & & & \\
\hline \multirow{2}{*}{ LA active emptying fraction } & Mean \pm SD & $42.85 \pm 10.05$ & $34.38 \pm 11.35$ & $34.40 \pm 10.78$ & \multirow{2}{*}{0.001} & \multirow{2}{*}{0.001} & \multirow{2}{*}{0.994} \\
\hline & Range & $19.4-61.5$ & $15-58.6$ & $16.6-58.6$ & & & \\
\hline
\end{tabular}

TABLE 7: Correlation between LA volumes and demographic parameters.

\begin{tabular}{lcccccccc}
\hline & \multicolumn{2}{c}{$V_{\text {min }}$} & \multicolumn{2}{c}{$V_{\max }$} & \multicolumn{2}{c}{$V_{\text {prea }}$} & \multicolumn{2}{c}{ LAVi } \\
& $r$ & $P$ value & $r$ & $P$ value & $r$ & $P$ value & $r$ \\
\hline Age & $0.374^{* *}$ & 0.001 & $0.449^{* *}$ & 0.001 & 0.124 & 0.178 & $0.247^{* *}$ \\
Duration of diabetes & -0.048 & 0.713 & -0.066 & 0.617 & 0.060 & 0.647 & -0.007 \\
Weight & 0.054 & 0.557 & $0.198^{*}$ & 0.030 & 0.086 & 0.348 & -0.099 & 0.958 \\
BMI & 0.082 & 0.372 & $0.281^{* *}$ & 0.002 & 0.023 & 0.800 & 0.017 & 0.856 \\
\hline
\end{tabular}

emptying fraction as compared to the control group $(P<0.05)$ with no differences regarding the LA total stroke volume and LA active stroke volume between both study groups $(P>0.05)$ while patients with type 2 diabetes showed a significantly larger LA total stroke volume and lower LA active emptying fraction $(P<0.05)$ with no difference regarding the LA active stroke volume $(P>0.05)$ when compared to control subjects(Table 6).

Patients with type 2 diabetes showed a significantly larger LA total stroke volume as compared to those with type 1 diabetes $(P<0.05)$ with no differences in LA active stroke volume and LA active emptying fraction $(P>0.05)$ (Table 7$)$. 
5.5. Correlation of Patient Characteristics with Left Atrial Volume at Different Phases. Age showed a strong correlation with the left atrial volume at different phases $\left(V_{\min }\right.$ and $\left.V_{\max }\right)$ and the left atrial volume indexed to body surface area (LAVi) $(P<0.001,0.001$, and 0.007 , respectively) but showed no correlation with $V_{\text {preA }}$ volume $(P$ value $=0.178)$.

The weight and body mass index (BMI) showed a strong correlation with $V_{\max }$ only $(P$ value $=0.030$ and 0.002 , respectively.

The duration of diabetes mellitus showed no correlation with the left atrial volume at any phase $\left(V_{\min }, V_{\max }\right.$, and $V_{\text {preA) }}$ and left atrial volume index (LAVi).

5.6. Correlation of 2D LA Volume, Area, and Dimensions with $3 D$ LA Volume. The maximum left atrial volume obtained by 2D echocardiography correlated positively with the phasic left atrial volume obtained by 3D echocardiography $\left(V_{\min }\right.$, $V_{\max }$, and $V_{\text {preA }}$ ).

The maximum left atrial volume indexed to the body surface area (LAVi) showed positive correlation with $V_{\min }$ and $V_{\max }$ with no correlation with $V_{\text {preA }}$ (Table 7).

The left atrial area (LA area) obtained by 2D echocardiography from apical four-chamber view showed a significantly positive correlation with $V_{\min }, V_{\max }$, and LAVi $(P$ value $<0.05)$.

The left atrial length (LA leng.) obtained by $2 \mathrm{D}$ echocardiography from apical four-chamber view showed a significantly positive correlation with $V_{\max }$ and LAVi $(P$ value $<$ 0.05).

The left atrial diameter (maximum diameter) obtained by 2D echocardiography with M-mode from parasternal longaxis view showed a strong correlation with $V_{\min }$ and $V_{\max }$ $(P$ value $<0.05)$ with no correlation with LAVi and $V_{\text {preA }}$ $(P$ value $>0.05)$.

The mitral inflow velocity $(E / A)$ ratio showed a significant correlation with $V_{\min }$ and $V_{\max }(P$ value $<0.05)$ (Table 8).

The regression analysis model demonstrated that the presence of diabetes mellitus, type of diabetes mellitus, and age were independent predictors of $V_{\min }$ while only the type of diabetes mellitus was an independent predictor of $V_{\max }$; it also demonstrated that the presence of diabetes and type of diabetes were independent predictors of LAVi. Only the type of diabetes was an independent factor of the LA total stroke volume.

\section{Discussion}

The first published report of 3D echocardiographic evaluation of the LA size was that of King et al. in 1992. The authors studied 30 patients who were referred to the echocardiography laboratories for clinically indicated examinations. The patients were chosen randomly and were unselected for the type of heart disease. Each patient was required to have a technically satisfactory study to be included. The study concluded the potential usefulness of 3D echocardiography in completing 2D echocardiographic data in a comprehensive assessment of the LA size [21].
As we evaluated LV dimensions, LV systolic function, LA length, and LA anteroposterior diameter using 2D echocardiography, we did not observe any significant difference when we compared type 2 diabetic patients with normal controls; our results were in agreement with Atas et al. in 2014, who showed no significant difference regarding LV dimensions, LV systolic function, LA length, and LA anteroposterior diameter by using $2 \mathrm{D}$ echocardiography, when they compared the type 2 diabetic group with the normal control group [22].

As we used 2D guidance Doppler wave to interrogate mitral inflow velocities, we observed that atrial filling $(A)$ wave velocity was $75.04 \pm 17.82 \mathrm{~cm} / \mathrm{sec}$ in type 2 diabetic patients which was significantly increased among type 2 diabetic patients when compared to normal controls, and we also observed that the $E / A$ velocity ratio was $0.94 \pm 0.30$ in type 2 diabetic patients which was decreased significantly among type 2 diabetic patients as compared to normal controls.

Gul and colleagues in (2009) compared diastolic parameters in 81 type 1 diabetic patients and 50 healthy volunteers using both pulse wave Doppler and tissue Doppler imaging to evaluate the possible effects of type 1 diabetes on left ventricular dysfunction; they demonstrated that the $(A)$ velocity was $63 \pm 0.30 \mathrm{~cm} / \mathrm{sec}$ and showed to be significantly increased in type 1 diabetic patients when compared with normal controls [23].

These results are similar to ours; in our study, the $(A)$ velocity was $73.83 \pm 11.74 \mathrm{~cm} / \mathrm{sec}$ in type 1 diabetic patients and was significantly increased when compared to normal controls.

AThere are several methods to measure the left atrial volume using 2DE, including Simpson's biplane method of discs, biplane area length, and the prolate ellipse. The two biplane methods compare closely and are recommended as the standard for left atrial volumes in ASE guidelines. The biplane method has the most accurate $2 \mathrm{D}$ echocardiographic estimation of LA volumes compared with CT, providing the closest approximation to the true left atrial volume [24].

The LA maximum volume $\left(V_{\max }\right)$ was evaluated by $2 \mathrm{D}$ echocardiography with different methods (4-chamber LA volume, 2-chamber LA volume, and biplane Simpson's method); we observed that the LA maximum volume was increased significantly in type 2 diabetic patients as compared to type 1 diabetic patients and normal controls by all methods of examinations used.

In 2014, Atas and colleagues examined 40 type 2 diabetics and 40 normal healthy controls using RT3DE in addition to conventional 2D echocardiography to assess the LA volume and phasic function and demonstrated that LA volumes, LA maximum volume $\left(V_{\max }\right)$ which was $40.9 \pm 11.9 \mathrm{ml}$, LA minimum volume $\left(V_{\min }\right)$ which was $15.6 \pm 5.9 \mathrm{ml}$, and LA total stroke volume which was $25.8 \pm 7.1 \mathrm{ml}$ were higher significantly in diabetic patients, while LA active emptying fraction was $38.5 \pm 13$ and was significantly reduced in type 2 diabetic patients, with no significant difference between groups regarding LA active stroke volume and LA volume before atrial contraction $\left(V_{\text {preA }}\right)$ [22].

Our results are in agreement with Atas et al. observations; as LA volumes in type 2 diabetic patients were higher 
TABLE 8: Correlation between 2D data and LA 3D volumes.

\begin{tabular}{lcccccccc}
\hline & \multicolumn{2}{c}{$V_{\text {min }}$} & \multicolumn{2}{c}{$V_{\max }$} & \multicolumn{2}{c}{$V_{\text {preA }}$} & \multicolumn{2}{c}{ LAVi } \\
& $r$ & $P$ value & $r$ & $P$ value & $r$ & $P$ value & 0.126 & $0.460^{* *}$ \\
\hline 2C LAV & $0.337^{* *}$ & 0.001 & $0.742^{* *}$ & 0.001 & 0.141 & 0.126 & $0.580^{* *}$ \\
2C (BiP) & $0.444^{* *}$ & 0.001 & $0.906^{* *}$ & 0.001 & 0.143 & 0.120 & 0.001 \\
LA volume & $0.469^{* *}$ & 0.001 & $0.764^{* *}$ & 0.001 & 0.131 & 0.154 & $0.515^{* *}$ & 0.001 \\
LA area & $0.333^{* *}$ & 0.001 & $0.615^{* *}$ & 0.001 & 0.116 & 0.207 & $0.386^{* *}$ & 0.001 \\
LA length & 0.172 & 0.060 & $0.413^{* *}$ & 0.001 & 0.172 & 0.061 & $0.292^{* *}$ & 0.001 \\
LA diameter & $0.246^{* *}$ & 0.007 & $0.465^{* *}$ & 0.001 & 0.111 & 0.228 & $0.230^{*}$ & 0.012 \\
E & -0.055 & 0.550 & -0.009 & 0.925 & 0.011 & 0.907 & 0.076 \\
$A$ & $0.181^{*}$ & 0.049 & 0.178 & 0.053 & -0.058 & 0.528 & 0.140 \\
E/A & $-0.183^{*}$ & 0.046 & $-0.182^{*}$ & 0.048 & -0.031 & 0.738 & -0.101 \\
\hline
\end{tabular}

significantly in type 2 diabetic patients when compared to normal controls; $V_{\max }$ was $44.09 \pm 9.44 \mathrm{ml}, V_{\min }$ was $17.29 \pm 4.11 \mathrm{ml}$, and total stroke volume was $25.26 \pm 4.14$ $\mathrm{ml}$; also, LA active emptying fraction was $34.40 \pm 10.78$ which was reduced significantly in type 2 diabetic patients. There was no difference between the groups regarding LA active stroke and $V_{\text {preA }}$.

Huang and colleagues in (2010) examined left atrial function in patients with type 2 diabetes mellitus (DM). Fiftyeight type $2 \mathrm{DM}$ patients as the DM group and forty healthy people as the normal control group were enrolled in their study. EUB-6500 echocardiographic imaging system with LA volume tracking was applied to display and analyze the LA volume curve imaging on LV apical two- and fourchamber views. They concluded that LA maximum volume indexed to the body surface area (LAVi) was significantly higher in the type 2 diabetic patients when compared to normal controls [5].

Our study showed that the LA maximum volume indexed to the body surface area (LAVi) was significantly larger $23.55 \pm 3.37 \mathrm{ml} / \mathrm{m}^{2}$ in type 2 diabetic patients than in normal controls which was $20.30 \pm 2.11 \mathrm{ml} / \mathrm{m}^{2}$, which is similar to the results of Huang et al. 2010, in spite of using RT3DE volume analysis in our study instead of the LA volume tracking method for evaluating LA volume.

Acar and colleagues in 2009 studied LA volume and function; 43 patients with type 1 diabetes mellitus and 42 controls were enrolled in their study; they concluded that LAVi, $V_{\max }$, $V \min , V_{\text {preA }}$, and LA total stroke volume were similar between the both groups. However, they observed a significant increase in LA active emptying fraction in their type 1 diabetic patients which was $45.7 \pm 8.6$ versus $36.3 \pm 11.1$ in normal controls [25].

In type 1 diabetic patients enrolled in our study, we observed that the LA maximum volume index, LA maximum volume, LA minimum volume, LA volume before atrial contraction, and LA total stroke volume were $21.23 \pm 4.29 \mathrm{ml} /$ $\mathrm{m}^{2}, 35.29 \pm 7.64 \mathrm{ml}, 12.78 \pm 2.12 \mathrm{ml}, 23.02 \pm 4.34 \mathrm{ml}$, and $22.91 \pm 4.88 \mathrm{ml}$, respectively, in type 1 diabetic patients versus $20.30 \pm 2.11 \mathrm{ml}, 32.44 \pm 6.81 \mathrm{ml}, 12.41 \pm 2.59 \mathrm{ml}, 23.68 \pm$ $6.16 \mathrm{ml}$, and $21.81 \pm 4.04 \mathrm{ml}$, respectively, in normal controls, with no significant differences between the two groups which was in line with Acar et al. observations.

On the other hand, we found that the left atrial pump function was impaired in our type 1 diabetic patients which was opposite to Acar et al. However, our type 1 diabetic patients displayed lower active emptying fraction and similar passive emptying fraction (LA volume before atrial contraction) values compared to controls which is not an expected finding for impaired left ventricular diastolic compliance in which a compensatory increase of LA contractility and pump function is expected. Based on these findings, it may be suggested that an independent atrial cardiomyopathy associated with diabetes might also be operative on the altered left atrial volume and functions in our diabetic patients [26].

In a study by Chillo and colleagues in 2013, they performed echocardiography for 122 type 2 and 58 type 1 diabetic patients to determine the prevalence of LA enlargement and its relation to LV diastolic dysfunction among asymptomatic diabetics and they concluded that enlarged LA volume and $\mathrm{LV}$ diastolic dysfunction were more common in type 2 than in type 1 diabetic patients. Patients with enlarged LAVi were older when compared with patients with normal LAVi both among type 1 and type 2 diabetic groups [27].

As we compared type 1 diabetics with type 2 diabetics, we observed that LA volumes were increased in type 2 diabetic patients, and diastolic dysfunction was more common among type 2 diabetic patients; however, both type 1 and type 2 diabetics showed significantly impaired LA pumping function. This finding may indicate deterioration of active relaxation and compliance and contractility of LA myocardium in diabetic patients. Although the mechanism of impairment is not clear, injury to atrial myocardium caused by sustained hyperglycemia and fibrotic alteration of LA have been suggested to be contributing factors; age and nature of disease may be the cause for the difference between the 2 types of diabetes.

When we compared the 2D echocardiographic LA area with $2 \mathrm{D}$ and 3D LA volume, there was a significantly positive correlation and insignificant difference; our results were explained by an abstract titled "Comparison of left atrial size by freehand scanning three-dimensional echocardiography 
and two-dimensional echocardiography" reported by Kawai and colleagues in 2004. The 2D LA area and 2D LA volume showed a significant positive correlation with the 3D LA volume [28].

The anteroposterior diameter of LA measured using 2Dguided M-mode from parasternal long-axis view showed no correlation with LA volume, and this was explained previously by Lester et al. LA dilatation might not be evenly distributed in all planes, and measurement of anteroposterior dimension is likely to be insensitive to changes in LA size [29].

In our diabetic patients, $80 \%$ of type 1 and $40 \%$ of type 2 diabetic patients were classified as having normal diastolic function $(E / A \geq 1)$; interestingly, LA enlargement was present in this group as well. These observations are similar to those reported by Jarnert et al. In that study, LAVi was increased even in the subgroup with normal diastolic function. This suggests that an altered LV diastolic function in DM only contributes in part to observed LA changes. Thus, it is likely that an independent atrial cardiomyopathy associated with DM may be a likely contributor to LA enlargement [30].

Zhong et al. performed a study using RT3DE in healthy subjects to calculate LAVi, LA volume maximum $\left(V_{\max }\right)$, and LA volume minimum $\left(V_{\min }\right)$ which increased with age. Similarly, we found that LAVi, $V_{\max }$, and $V_{\min }$ were positively correlated with age [31].

Gardts and colleagues in 2002 assessed the left atrial volume in 941 hypertensive patients with a mean age 66 years using 2D echocardiography; they concluded that the LA volume was positively correlated with body mass index and age [32].

The present study was designed to assess the effects of diabetes mellitus (type 1 and type 2) on left atrial size and function and showed that the LA volume was increased and LA mechanical function was impaired in type 2 diabetic patients, whereas type 1 diabetic patients only showed impaired LA pump function.

\section{Study Limitations}

This was a cross-sectional study, and the prognostic importance of our findings is not clear. The sample size was small, and further studies on a larger number of patients are needed.

The LA appendage has an important role for the function and volume measurements of LA, but it was excluded in the study which may have affected the results.

Software Q Lab Philips version 6 was used for the analysis of $3 \mathrm{D}$ volumetric data which is a relatively old version of the available software and is originally designed for evaluation of left ventricular volumes. However, using this software for evaluation of LA volumes also seems to be prudent as it has been used by many other studies in the literature. As to our knowledge, there is no dedicated LA software available in the market.

\section{Conclusion}

(1) Evaluation of asymptomatic diabetic patients by using RT3DE atrial volume analysis may facilitate recognition of subtle alterations related with type 1 and type 2 diabetes

(2) Patients with type 2 diabetes mellitus have increased left atrial volume and impaired left atrial compliance and contractility in relation to normal healthy subjects

(3) Patients with type 1 diabetes mellitus have impaired left atrial contractility in relation to normal healthy subjects

(4) The presence of diabetes mellitus, type of diabetes mellitus, and age were independent predictors of $V_{\text {min }}$ while only the type of diabetes mellitus was the independent predictor of $V_{\max }$

(5) The presence of diabetes mellitus and type of diabetes mellitus were independent predictors of LAVi, and only the type of diabetes mellitus was the independent predictor of LA total stroke volume while none of them were independent predictors of LA active emptying fraction

(6) Intrinsic alterations in atrial myocardial activity seem to be responsible for left atrial dysfunction in addition to impairment in left ventricular diastolic function which is known to be common in diabetic patients

\section{Abbreviations}

2c LAV: 2-chamber left atrial volume

2DE: Two-dimensional echocardiography

3D: Three-dimensional echocardiography

4c LAV: 4-chamber left atrial volume

AEF: Active emptying fraction

AEV: $\quad$ Active emptying volume

ASV: $\quad$ Active stroke volume

CCT: Cardiac computed tomography

CHD: Coronary heart disease

CHF: $\quad$ Congestive heart failure

CMR: Cardiac magnetic resonance

CT: $\quad$ Computed tomography

CVD: Cardiovascular disease

D1 group: Type 1 diabetic group

D2 group: Type 2 diabetic group

DBP: $\quad$ Diastolic blood pressure

DCM: Dilated cardiomyopathy

DD: Diastolic dysfunction

DM: Diabetes mellitus

$E / A$ : $\quad$ Ratio of peak early to peak late mitral inflow velocity

E/E: $\quad$ Ratio of peak early mitral inflow to peak early diastolic tissue velocity of mitral annulus

EDV: $\quad$ End diastolic volume

HbA1C: Gylcated hemoglobin

HDL-C: High-density lipoprotein cholesterol

HF: $\quad$ Heart failure

LA: $\quad$ Left atrium

LAA: $\quad$ Left atrial appendage 
LAAEV: Left atrial active emptying volume

LAEF: Left atrial ACTIVE emptying fraction

LAFV: Left atrial filling volume

LAPEV: Left atrial passive emptying volume

LASV: Left atrial total stroke volume

LAV: Left atrial volume

LAVi: Left atrial volume index

LDL: $\quad$ Low-density lipoprotein

LS: $\quad$ Longitudinal strain

LV: $\quad$ Left ventricle

LVEDd: Left ventricular end diastolic dimension

LVEF: Left ventricular ejection fraction

LVESd: Left ventricular end systolic dimension

LVM: $\quad$ Left ventricular mass

PALS: $\quad$ Peak atrial longitudinal strain

ROS: $\quad$ Reactive oxygen species

RT3DE: Real-time three-dimensional echocardiography

S: $\quad$ Seconds

SBP: $\quad$ Systolic blood pressure

SMI: Asymptomatic myocardial infarction

SR: $\quad$ Strain rate

SR-B: $\quad$ Scavengener receptor-B

SRI: $\quad$ Strain rate imaging

STE: $\quad$ Speckle-tracking echocardiography

TDI: $\quad$ Tissue Doppler imaging

TG: $\quad$ Triglycerides

TOE: Transesophageal echocardiography

TPA: $\quad$ Tissue plasminogen activator

TTE: Transthoracic echocardiography

$V_{\text {max }}: \quad$ Left atrial maximum volume

$V_{\min }: \quad$ Left atrial minimum volume

$V_{\text {preA }}: \quad$ Left atrial volume before atrial contraction.

\section{Data Availability}

The data used to support the findings of this study are available from the corresponding author upon request.

\section{Disclosure}

I confirm that I have read, understand, and agreed to the submission guidelines, policies, and submission declaration of the journal. I confirm that all authors of the manuscript have no conflict of interests to declare. I confirm that the manuscript is the authors' original work, and the manuscript has not received prior publication and is not under consideration for publication elsewhere. I confirm that all authors listed on the title page have contributed significantly to the work, read the manuscript, attested to the validity and legitimacy of the data and its interpretation, and agreed to its submission. I confirm that the paper now submitted is not copied or a plagiarized version of some other published work. I declare that I shall not submit the paper for publication in any other Journal or Magazine till the decision is made by journal editors. I confirm that we did not receive any fund from any source or organization.

\section{Conflicts of Interest}

The authors declare that they have no conflicts of interest.

\section{References}

[1] R. M. Lang, L. P. Badano, V. Mor-Avi et al., "Recommendations for cardiac chamber quantification by echocardiography in adults: an update from the American Society of Echocardiography and the European Association of Cardiovascular Imaging," Journal of the American Society of Echocardiography, vol. 28, no. 1, pp. 1-39.e14, 2015.

[2] A. C. Y. To, S. D. Flamm, T. H. Marwick, and A. L. Klein, "Clinical utility of multimodality LA imaging: assessment of size, function, and structure," JACC: Cardiovascular Imaging, vol. 4, no. 7, pp. 788-798, 2011.

[3] E. Aune, M. Baekkevar, J. Roislien, O. Rodevand, and J. E. Otterstad, "Normal reference ranges for left and right atrial volume indexes and ejection fractions obtained with realtime three-dimensional echocardiography," European Journal of Echocardiography, vol. 10, no. 6, pp. 738-744, 2009.

[4] D. Y. Leung, A. Boyd, A. A. Ng, C. Chi, and L. Thomas, "Echocardiographic evaluation of left atrial size and function: current understanding, pathophysiologic correlates, and prognostic implications," American Heart Journal, vol. 156, no. 6, pp. 1056-1064, 2008.

[5] G. Huang, L. Zhang, M. Xie, M. Fu, J. Huang, and Q. Lv, "Assessment of left atrial function in diabetes mellitus by left atrial volume tracking method," Journal of Huazhong University of Science and Technology [Medical Sciences], vol. 30, no. 6, pp. 819-823, 2010.

[6] S. Y. Li, L. Zhang, B. W. Zhao et al., "Two-dimensional tissue tracking: a novel echocardiographic technique to measure left atrial volume: comparison with biplane area length method and real time three-dimensional echocardiography," Echocardiography, vol. 31, no. 6, pp. 716-726, 2014.

[7] W. Oliveira, O. Campos, F. Cintra et al., "Impact of continuous positive airway pressure treatment on left atrial volume and function in patients with obstructive sleep apnoea assessed by real-time three-dimensional echocardiography," Heart, vol. 95, no. 22, pp. 1872-1878, 2009.

[8] S.-W. Zhong, Y.-Q. Zhang, L.-J. Chen, S.-S. Wang, and W.-H. Li, "Evaluation of left ventricular volumes and function by real time three-dimensional echocardiography in children with functional single left ventricle: a comparison between QLAB and TomTec," Echocardiography, vol. 32, no. 10, pp. 1554-1563, 2015.

[9] M. Rosca, P. Lancellotti, B. A. Popescu, and L. A. Pierard, "Left atrial function: pathophysiology, echocardiographic assessment, and clinical applications," Heart, vol. 97, no. 23, pp. 1982-1989, 2011.

[10] B. Khankirawatana, S. Khankirawatana, and T. Porter, "How should left atrial size be reported? Comparative assessment with use of multiple echocardiographic methods," American Heart Journal, vol. 147, no. 2, pp. 369-374, 2004.

[11] R. Ancona, S. Comenale Pinto, P. Caso et al., "Left Atrium by Echocardiography in Clinical Practice: From Conventional Methods to New Echocardiographic Techniques," The Scientific World Journal, vol. 2014, Article ID 451042, 15 pages, 2014.

[12] J. L. Dorosz, D. C. Lezotte, D. A. Weitzenkamp, L. A. Allen, and E. E. Salcedo, "Performance of 3-dimensional echocardiography in measuring left ventricular volumes and ejection fraction: a systematic review and meta-analysis," Journal of the American College of Cardiology, vol. 59, no. 20, pp. 1799$1808,2012$. 
[13] A. C. Y. To, S. D. Flamm, T. H. Marwick, and A. L. Klein, "Clinical Utility of Multimodality LA Imaging," JACC: Cardiovascular Imaging, vol. 4, no. 7, pp. 788-798, 2011.

[14] A. M. Anwar, O. I. I. Soliman, M. L. Geleijnse, A. Nemes, W. B. Vletter, and F. J. ten Cate, "Assessment of left atrial volume and function by real-time three-dimensional echocardiography," International Journal of Cardiology, vol. 123, no. 2, pp. 155-161, 2008.

[15] F. J. N. Mancuso, V. A. Moisés, D. R. Almeida et al., "Left atrial volume determinants in patients with non-ischemic dilated cardiomyopathy," Arquivos Brasileiros de Cardiologia, vol. 105, no. 1, pp. 65-70, 2015.

[16] Y. Miyasaka, S. Tsujimoto, H. Maeba et al., "Left atrial volume by real-time three-dimensional echocardiography: validation by 64-slice multidetector computed tomography," Journal of the American Society of Echocardiography, vol. 24, no. 6, pp. 680-686, 2011.

[17] A. Rohner, M. Brinkert, N. Kawel et al., "Functional assessment of the left atrium by real-time three-dimensional echocardiography using a novel dedicated analysis tool: initial validation studies in comparison with computed tomography," European Journal of Echocardiography, vol. 12, no. 7, pp. 497-505, 2011.

[18] C. Russo, Z. Jin, R. Liu et al., "LA Volumes and Reservoir Function Are Associated With Subclinical Cerebrovascular Disease," JACC: Cardiovascular Imaging, vol. 6, no. 3, pp. 313-323, 2013.

[19] R. M. Lang, M. Bierig, R. B. Devereux et al., "Recommendations for chamber quantification: a report from the American Society of Echocardiography's Guidelines and Standards Committee and the Chamber Quantification Writing Group, developed in conjunction with the European Association of Echocardiography, a branch of the European Society of Cardiology," Journal of the American Society of Echocardiography, vol. 18, no. 12, pp. 1440-1463, 2005.

[20] S. Lupu, A. Mitre, and D. Dobreanu, "Left atrium function assessment by echocardiography, physiological and clinical implications," Medical Ultrasonography, vol. 16, no. 2, pp. 152-159, 2014.

[21] D. L. King, M. R. Harrison, D. L. King Jr., A. S. Gopal, R. P. Martin, and A. N. DeMaria, "Improved reproducibility of left atrial and left ventricular measurements by guided threedimensional echocardiography," Journal of the American College of Cardiology, vol. 20, no. 5, pp. 1238-1245, 1992.

[22] H. Atas, A. Kepez, D. B. Atas et al., "Effects of diabetes mellitus on left atrial volume and functions in normotensive patients without symptomatic cardiovascular disease," Journal of Diabetes and Its Complications, vol. 28, no. 6, pp. 858-862, 2014.

[23] K. Gul, A. S. Celebi, F. Kacmaz et al., "Tissue Doppler imaging must be performed to detect early left ventricular dysfunction in patients with type 1 diabetes mellitus," European Journal of Echocardiography, vol. 10, no. 7, pp. 841-846, 2009.

[24] R. R. Buechel, F. P. Stephan, G. Sommer, J. Bremerich, M. J. Zellweger, and B. A. Kaufmann, "Head-to-head comparison of Two-Dimensional and three-dimensional echocardiographic methods for left atrial chamber quantification with magnetic resonance imaging," Journal of the American Society of Echocardiography, vol. 26, no. 4, pp. 428-435, 2013.

[25] G. Acar, A. Akcay, A. Sokmen et al., "Assessment of atrial electromechanical delay, diastolic functions, and left atrial mechanical functions in patients with type 1 diabetes melli- tus," Journal of the American Society of Echocardiography, vol. 22, no. 6, pp. 732-738, 2009.

[26] J. Asbun and F. J. Villarreal, "The pathogenesis of myocardial fibrosis in the setting of diabetic cardiomyopathy," Journal of the American College of Cardiology, vol. 47, no. 4, pp. 693700, 2006

[27] P. Chillo, Å. E. Rieck, J. Lwakatare, J. Lutale, and E. Gerdts, "Left atrial volume index as a marker of left ventricular diastolic dysfunction in asymptomatic Tanzanian diabetic patients," Blood Pressure, vol. 22, no. 2, pp. 86-93, 2012.

[28] J. Kawai, K. Tanabe, C. Wang et al., "Comparison of left atrial size by freehand scanning three-dimensional echocardiography and two-dimensional echocardiography," European Journal of Echocardiography, vol. 5, no. 1, pp. 18-24, 2004.

[29] S. J. Lester, E. W. Ryan, N. B. Schiller, and E. Foster, "Best method in clinical practice and in research studies to determine left atrial size," The American Journal of Cardiology, vol. 84, no. 7, pp. 829-832, 1999.

[30] C. Jarnert, A. Melcher, K. Caidahl, H. Persson, L. Rydén, and M. J. Eriksson, "Left atrial velocity vector imaging for the detection and quantification of left ventricular diastolic function in type 2 diabetes," European Journal of Heart Failure, vol. 10, no. 11, pp. 1080-1087, 2008.

[31] L. Zhong, L. K. Tan, C. J. Finn, D. Ghista, R. Liew, and Z. P. Ding, "Effects of age and gender on left atrial ejection force and volume from real-time three-dimensional echocardiography," Annals of the Academy of Medicine, Singapore, vol. 41, pp. 161-169, 2012.

[32] E. Gerdts, L. Oikarinen, V. Palmieri et al., "Correlates of left atrial size in hypertensive patients with left ventricular hypertrophy," Hypertension, vol. 39, no. 3, pp. 739-743, 2002.

[33] C. Aggeli, I. Felekos, S. Kastellanos et al., "Real-time threedimensional echocardiography: never before clinical efficacy looked so picturesque," International Journal of Cardiology, vol. 198, pp. 15-21, 2015. 